\title{
IMPEDANCE BUDGET FOR THE KEK B-FACTORY
}

\author{
Y. H. Chin and K. Satoh, KEK, 1-1 Oho, Tsukuba-shi, Ibaraki-ken, 305, Japan
}

\begin{abstract}
In this paper, we deal with impedances of various beamline elements in the KEK B-Factory. We will also discuss the power deposition generated by a beam in the form of the higher-ordermode (HOM) losses by interacting with its surroundings.
\end{abstract}

\section{INTRODUCTION}

The KEK B-Factory (KEKB) is an electron-positron collider with unequal beam energies ( 8 and $3.5 \mathrm{GeV}$ ) for study of $\mathrm{B}$ meson physics[1]. The dominant issues in the KEKB in terms of beam instabilities and impedance are the very high beam current (2.6 $\mathrm{A}$ in the low energy ring (LER) and 1.1 $\mathrm{A}$ in the high energy ring (HER)) to achieve the high luminosity of $10^{34} \mathrm{~cm}^{-2} \mathrm{~s}^{-1}$, and a short bunch $\left(\sigma_{\mathrm{z}}=4 \mathrm{~mm}\right)$ to avoid a degradation of the luminosity by the hour-glass effect. Since the charges are distributed over many (5120) bunches, the bunch current is not unusually high. As a consequence, single-bunch effects are expected to be relatively moderate: their stability limits are beyond the design values with comfortable margins. The main concern, in turn, is on coupledbunch instabilities due to high-Q resonant structures such as RF cavities and the transverse resistive-wall instability at very low frequency (lower than the revolution frequency). The short bunch can pick up the impedance at very high frequency $(f \sim 20 \mathrm{GHz})$ and thus may create an enormous heat deposition by the higherorder mode (HOM) losses. This heating problem requires serious efforts to reduce the impedance of various beam components or eliminate candidates for trapping modes all together.

\section{IMPEDANCE OF COMPONENTS}

In this section, we summarize our estimate of impedances and loss factors of various beamline components. Among impedance-generating elements of the rings, the largest contributors are RF cavities, the resistive wall, masks at arc, pumping slots and bellows (because of their large number).

\section{A. RF cavities}

Two types of RF cavities are under consideration for the KEKB. The ARES (Accelerator Resonantly coupled with Energy Storage) cavity is a normal-conductive cavity with an energy storage cavity to reduce the detuning of the accelerating frequency due to a large beam loading effect. Another candidate is a superconductive cavity with HOM absorbers made of ferrite material attached to the beam pipe. Although a final decision on which cavity will be employed in the KEKB has not yet been made, we only deal with the impedance and loss factor of the ARES cavity in this paper. The beam power of 5.2 MW will be provided by 20 and 40 cells of the ARES cavities in the LER (with wiggler) and HER, respectively. The impedance of the HOMs in the ARES have been calculated using a computer code MAFIA. Most HOMs are significantly de-Qed, typically much less than 100, by the HOM damper consisting of a coaxial wave guide equipped with a notch filter. Using the program ABCI[2], we have estimated that the main body of the ARES cavity produces a loss factor of $0.529 \mathrm{~V} / \mathrm{pC}$ at bunch length $\sigma_{\mathrm{z}}=4 \mathrm{~mm}$. If this cavity is connected to the beam chamber (radius $=50 \mathrm{~mm}$ ) at both ends with $100 \mathrm{~mm}$ long tapers, the additional loss factor will be $0.363 \mathrm{~V} / \mathrm{pC}$. In total, the loss factor of one cell of the ARES cavity is $0.892 \mathrm{~V} / \mathrm{pC}$.

\section{B. Resistive-wall}

The material of the KEKB beam chamber was chosen to be copper because of its low photon-induced gas desorption coefficient, its high thermal conductivity, and its large photon absorption coefficient. Its high electrical conductivity also helps to reduce the resistive-wall impedance. Nevertheless, this is still the dominant source of transverse impedance for the coupled-bunch instability. The total transverse resistive-wall impedance of the circular pipe with an inner radius $b$ is given by

$$
Z_{\mathrm{RW}}(\omega)=Z_{0}(\operatorname{sgn}(\omega)-i) \frac{\delta R}{b^{3}},
$$

where $Z_{0}(\cong 377 \Omega)$ is the characteristic impedance of vacuum, $\delta$ is the skin depth, $R$ is the average radius of the ring, and $\operatorname{sgn}(\omega)$ denotes the sign of $\omega$. For the LER vacuum chamber $(b=50$ $\mathrm{mm}$ ), Eq. (1) gives the resistive-wall impedance of $0.3 \mathrm{M} \Omega / \mathrm{m}$ at the revolution frequency $100 \mathrm{kHz}$, while the impedance decreases to $2 \mathrm{k} \Omega / \mathrm{m}$ at the cutoff frequency $2.3 \mathrm{GHz}$ of the chamber. The HER chamber of racetrack shape may be approximated by a circular one with a diameter of $25 \mathrm{~mm}$.

\section{Masks at arc}

Each bellows has a mask ( $5 \mathrm{~mm}$ high) located in its front to be shielded from the synchrotron radiation from a nearby bending magnet. There are about 1000 bellows (one bellows on both sides of each quadrupole magnet. There will be no mask for BPMs). The cross section of the mask in the medium plane is shown in Fig. 1. For accurate calculations of wake potentials and loss factors, a 3-D program MASK30 has been developed which solves the Maxwell equations directly in time domain. Using this code, we have found that the total longitudinal impedance of 1000 masks is

$$
\operatorname{Im}\left[\frac{Z(\omega)}{n}\right]=2.8 \times 10^{-3} \Omega,
$$

where $n$ expresses the frequency $\omega$ divided by the revolution frequency $\omega_{0}, n=\omega / \omega_{0}$. The total loss factor is

$$
k_{\mathrm{L}}=4.6 \mathrm{~V} / \mathrm{pC},
$$

which corresponds the total HOM power of $62 \mathrm{~kW}$ in the LER.

\section{Pumping slots}

The current design of pumping slots adopts the so-called "hidden holes" structure similar to those of HERA and PEP-II. A slot 


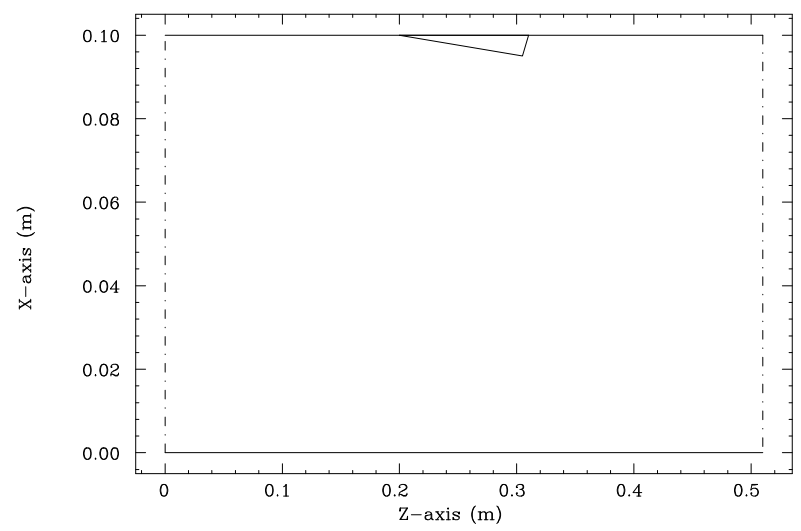

Figure. 1. Mask at arc

has a rectangular shape with rounded edges, which is long in the beam-axis direction (100 $\mathrm{mm}$ long, $4 \mathrm{~mm}$ wide). The slot is patched on the pumping chamber side by a rectangular grid. They help to prevent the microwave power generated somewhere else from penetrating through the slots to the pumping chamber and then depositing the energy in the NEG pumps. There are analytic formulae for impedance and loss factor of such a narrow slot by Kurennoy[3]. We found that the total inductive impedance of the pumping slots (there are 8 slots per meter over the total length of $1800 \mathrm{~m}$ ) with thickness correction is

$$
\operatorname{Im}\left[\frac{Z(\omega)}{n}\right]=0.8 \times 10^{-3} \Omega .
$$

The total loss factor was calculated to be

$$
k_{\mathrm{L}}=10.6 \mathrm{~V} / \mathrm{pC} \text {. }
$$

\section{E. $B P M s$}

The annular gap (or groove) in a BPM between the button electrode and the supporting beam chamber can be approximated by a regular octagon. The impedance of a BPM can be thus calculated from the same formula for a narrow slot considering it as a combination of eight narrow slots (two transverse, two longitudinal, and four tilted)[4]. If we neglect small contributions from the longitudinal slots, and consider four tilted slots as two transverse ones, the impedance of the BPM becomes equivalent to that of the four transverse slots. For 400 four-button BPMs (radius $=6.5 \mathrm{~mm}$, gap $=1 \mathrm{~mm}$, and thickness $=1 \mathrm{~mm}$ ), the total inductive impedance is

$$
\operatorname{Im}\left[\frac{Z(\omega)}{n}\right]=1.3 \times 10^{-4} \Omega .
$$

The total loss factor of BPMs is

$$
k_{\mathrm{L}}=0.71 \mathrm{~V} / \mathrm{pC} \text {. }
$$

\section{F. Mask at IP}

There are four masks (two large and two small) on both side of the beryllium chamber at the interaction point (IP) to shield it from the direct synchrotron radiation. Figure 2 shows their geometry. The loss factor due to these masks has been calculated using the code MASK30 and was found to be

$$
k_{\mathrm{L}}=5.2 \times 10^{-2} \mathrm{~V} / \mathrm{pC} \text {. }
$$

This value is about one-fourth of that obtained by ABCI using the axis-symmetrical model. The ratio of the two loss factors coincides with the ratio of the opening angle of the IP mask from the beam axis (about 90 degrees) to that of the entire circle. Based on this observation, we have learned that we can make a rough estimate of the loss factor for a 3-D structure by multiplying the opening angle ratio to a result for its axis-symmetrical model.

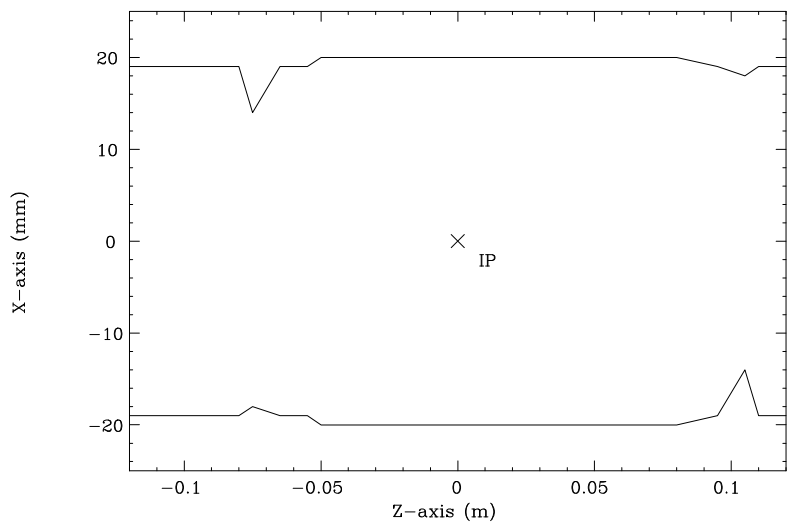

Figure. 2. Mask at the IP

Not all of the power generated at the IP will be deposited there. It depends on Q-values of modes excited between the masks. The beam chamber at the IP has the cutoff frequency at $6.04 \mathrm{GHz}$, and the tips of the taller masks creates another cutoff frequency at $8.202 \mathrm{GHz}$. If the wake fields between these two frequencies are trapped, the deposited energy will be (using ABCI and the translation law)

$$
k_{\mathrm{L}} \approx 0.1 \times \frac{1}{4} \mathrm{~V} / \mathrm{pC}=0.025 \mathrm{~V} / \mathrm{pC} .
$$

The corresponding power deposition at the IP in the LER is $\mathrm{P}=343 \mathrm{~W}$, which is $70 \%$ more than the design tolerance of 200 $\mathrm{W}$ for the beryllium window. However, if one takes a close look at the impedance of these modes, all the modes except the lowest one near $6.04 \mathrm{GHz}$ have Q-values much smaller than the one determined by the finite conductivity of the beryllium window. This is because the radius of the beam chamber remains the same inside and outside of the IP region separated by the masks, and thus the modes can escape to the outside region by making a bridge over the masks. The only trapped mode at $6.04 \mathrm{GHz}$ has a loss factor of $0.003 \mathrm{~V} / \mathrm{pC}$ and will deposit $41 \mathrm{~W}$ of the power at the IP. This amount of power deposition is acceptable provided that the frequency of the trapped mode is carefully detuned so that it will not resonate with the bunch frequency. The actual 3-D masks at the IP has a more open structure than the axissymmetrical model, and thus the power deposition might be even smaller.

\section{G. IR chamber}

The experimental chamber at the IR makes two large shallow tapers. Its impedance has been calculated using ABCI and found to be mostly inductive. They are

$$
\operatorname{Im}\left[\frac{Z(\omega)}{n}\right]=1.0 \times 10^{-3} \Omega .
$$


The loss factor without the contribution from the IP masks is

$$
k_{\mathrm{L}}=2.94 \times 10^{-1} \mathrm{~V} / \mathrm{pC},
$$

which corresponds to the HOM power loss of $3 \mathrm{~kW}$. This power deposition must be taken care of by e.g., putting an absorber in the chambers.

\section{H. Y-shaped recombination chambers}

The LER and HER chambers are combined to a single chamber on both sides of the IP (about $3 \mathrm{~m}$ away). The impedance and loss factor of two recombination chambers were modeled as axi-symmetrical structures, and the results by ABCI then were averaged proportional to the azimuthal filling factors. That gives a large loss factor almost equivalent to that of two ARES cavities:

$$
k_{\mathrm{L}}=1.6 \mathrm{~V} / \mathrm{pC} \text {. }
$$

\section{Bellows}

As explained in the subsection for the masks at arc, there are about 1,000 shielded bellows in both rings (one bellows on both sides of every quadrupole). We have adopted so called slidingfinger structure for bellows. These bellows produce predominantly inductive impedance. Their impedance has been calculated with use of ABCI. The imaginary part of the total impedance and the total loss factor for 1,000 bellows in the LER ring are

$$
\operatorname{Im}\left[\frac{Z(\omega)}{n}\right]=4.23 \times 10^{-3} \Omega
$$

and

$$
k_{\mathrm{L}}=2.5 \mathrm{~V} / \mathrm{pC} \text {. }
$$

Additional impedance is generated by the slits between the sliding fingers of the bellows. Using the same formula for a narrow slot, we found that their contributions are negligible.

\section{IMPEDANCE BUDGET}

The inductive impedances and the loss factors of the individual elements in the LER are tabulated in Table I. The total longitudinal wake potential for the LER is plotted in Fig. 3. The total HOM power deposition in the LER (corresponding to the loss factor of $42.2 \mathrm{~V} / \mathrm{pC}$ ) is $\mathrm{P}=570 \mathrm{~kW}$. In the HER, the total inductive impedance would be comparable to that of the LER. The total loss factor in the HER is larger than that of the LER by 18 $\mathrm{V} / \mathrm{pC}$ due to additional $20 \mathrm{RF}$ cavities, leading to $60 \mathrm{~V} / \mathrm{pC}$. The corresponding total HOM power deposition is $150 \mathrm{~kW}$. These numbers should be used in designing RF parameters.

\section{References}

[1] The KEKB Design Report in preparation.

[2] Y. H. Chin, Users's Guide for ABCI Version 8.8, CERN SL/94-02(AP) and LBL-35258 (1994).

[3] S. Kurennoy and Y. H. Chin, KEK Preprint 94-193 (1995). To be submitted to Part. Accelerators.

[4] S. Kurennoy, Report SSCL-636, Dallas (1993).

Table I

LER inductive impedance and loss factor budgets.

\begin{tabular}{lcc}
\hline Component & $\begin{array}{c}\text { Inductive impedance } \\
\operatorname{Im}[\mathrm{Z} / \mathrm{n}](\Omega)\end{array}$ & $\begin{array}{c}\text { Loss factor } \\
(\mathrm{V} / \mathrm{pC})\end{array}$ \\
\hline Cavities & - & 17.84 \\
Resistive-wall & $5.2 \times 10^{-3}$ at $2.3 \mathrm{GHz}$ & 4.0 \\
Masks at arc & $2.8 \times 10^{-3}$ & 4.6 \\
Pumping slots & $8.0 \times 10^{-4}$ & 10.6 \\
BPMs & $1.3 \times 10^{-4}$ & 0.71 \\
Mask at IP & negligible & $5.2 \times 10^{-2}$ \\
IR chamber & $1.0 \times 10^{-3}$ & $2.9 \times 10^{-1}$ \\
Y-chambers & $-8.0 \times 10^{-4}$ & 1.6 \\
Bellows & $4.23 \times 10^{-3}$ & 2.5 \\
\hline Total & 0.014 & 42.2 \\
\hline
\end{tabular}

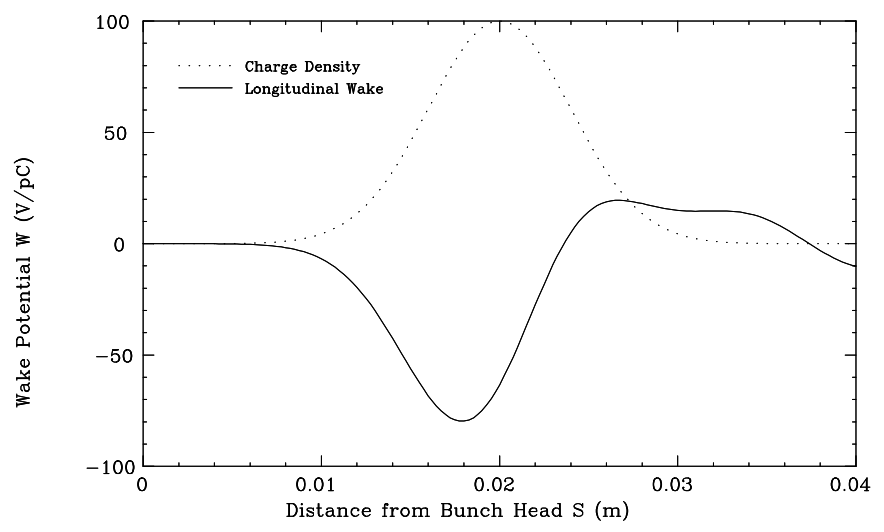

Figure. 3. Total longitudinal wake potential for the KEKB LER. 